\title{
Influenza vaccination recording system in Belgium
}

by

\author{
Antoine $\mathrm{J}^{1}$, Dupont $\mathrm{Y}^{1}$, Reynders $\mathrm{D}^{2}$, Van Laethem $\mathrm{Y}^{3}$, Van Ranst $\mathrm{M}^{4}$, \\ Neels $\mathrm{P}^{5}$, Roisin $\mathrm{T}^{5}$, Hamdani $\mathrm{J}^{5}$, Wuillaume $\mathrm{F}^{1}$
}

\section{Introduction}

Vaccination against influenza is one of the most effective measures to protect people from contracting illness during influenza epidemics and pandemics (1). In Belgium, the Interministerial Influenza Coordination Committee defined three main objectives for the vaccination campaign against Influenza $\mathrm{A}(\mathrm{H} 1 \mathrm{~N} 1) 2009$ pandemic. The first objective was to maintain the functioning of the health care system. The staff in hospitals and health professionals was then considered as priority groups for vaccination. This could limit the transmission of the infection from professional health workers to patients. The second objective was to limit complications and mortality due to influenza. Chronic conditions possibly related to a higher rate of complications and mortality have been listed in the literature available at the time. Patients suffering from one of those affections, women in their second or third trimester of pregnancy and parents of children aged less than six months constituted the second group to be vaccinated. The third objective was to maintain the continuity of the educational system and avoid an amplifying effect in educational institutions. The school staff was therefore defined as the third group to be vaccinated $(1,4)$.

Pandemrix (via a 10 doses phial packaging) was the only vaccine brand selected for the Belgian vaccination campaign. Pandemrix is an adjuvanted inactivated vaccine, containing antigens analogous to the A/California/7/2009(H1N1)v virus. The Influenza Scientific Committee recommended a single dose for persons aged 10 years or older, and two times half a dose for children aged 6 months to 9 years $(1,2,3)$.

The vaccination campaign was entrusted to general practitioners and hospitals, and the distribution of the vaccine was organised through the pharmaceutical network.

Simultaneously, a secure web-based tool was developed to allow practitioners to record data about patients they vaccinated. This recording system combined objectives of pharmacovigilance and evaluation of the vaccination campaign. Post-vaccine complications were recorded through a dedicated form used in the pharmacovigilance surveillance system managed by the Federal Agency for Medicines and Health Products (FAMHP). Patient data allowed the

\footnotetext{
Scientific Institute of Public Health, Directorate Public Health and Surveillance, Brussels, Belgium

2 FPS Health, Food Chain Safety and Environment, Brussels, Belgium

3 CHU Saint-Pierre, Department of Infectious Diseases, Brussels, Belgium

4 Rega Institute for Medical Research, University of Leuven, Leuven, Belgium

5 Federal Agency for Medicines and Health Products, Brussels, Belgium

jerome.antoine@wiv-isp.be
} 
Scientific Institute of Public Health (WIV-ISP) to describe the geographical and age distribution of vaccinated persons.

From 19 October until 6 November, the vaccine was available for hospital health workers only. From 7 November, the vaccine was proposed for free to other priority groups previously mentioned. The phasing of the vaccine distribution was also related to stock availability.

This article describes the web-based recording system and gives some results of the data collected.

\section{Material and methods}

The 'eH1N1 vaccination' web page was hosted on the eHealth platform which is a public portal created to promote and facilitate secure and electronic exchange of health data (5).

To access or encode data on this platform, an identity card reader, a Belgian electronic identity card and a PIN code (access code) were required. The 'eH1N1 vaccination' web page was available for two different groups: for citizens who wanted to check their vaccination status and print a vaccination certificate and for practitioners who wanted to check the vaccination status of a patient or record a new vaccination.

Data required for a new vaccination record were the national number of the patient (NISS code), the date of the vaccination and codes of the antigen and the adjuvant batches. No data on the risk status of the patient were recorded. For foreign patients without a NISS code, personal data (name, first name, birth year and sex) have to be encoded manually to print the certificate but these data were not stored in the database. Several control rules were installed to reduce coding mistakes to a minimum. Based on the national number, personal data of the patient (birth year, gender and postcode) were then extracted from the national database. An update of this information was sent by e-mail to the WIV-ISP every day but for confidentiality reasons, the national number of the patient was encrypted. These data were analysed using Stata 10.1 and a daily report was produced for the public health authorities as well as a weekly report for the FAMHP.

For the pharmacovigilance surveillance, a pandemic dedicated form used to record postvaccine adverse reactions was sent to practitioners through a medical newspaper (Folia Parmacotherapeutica). Patients with complications were welcome to contact their practitioner in order to fill out this form. This specific notification form was also accessible via the FAMHP website.

\section{Results}

Number of recorded vaccinations

Figure 1 shows the daily number of vaccinations recorded through the system and the weekly incidence curve of influenza-like illnesses (ILIs) as well as the epidemic threshold. 
The total number of vaccinations recorded from 19 October 2009 until 31 March 2010 amounted to 733,025 . On a weekly basis, $43 \%$ of the total number of vaccinations were recorded in week 46 (from 9 to 15 November), two weeks after the epidemic peak.

After week 46, this number reduced by $50 \%$ each week until the end of 2009 . In 2010 , less than 3000 vaccinations were recorded. To give a global idea, health authorities estimated that $2,000,000$ doses of the vaccine have been distributed during the pandemic period.

Figure 1. Daily number of vaccinated persons recorded through the system

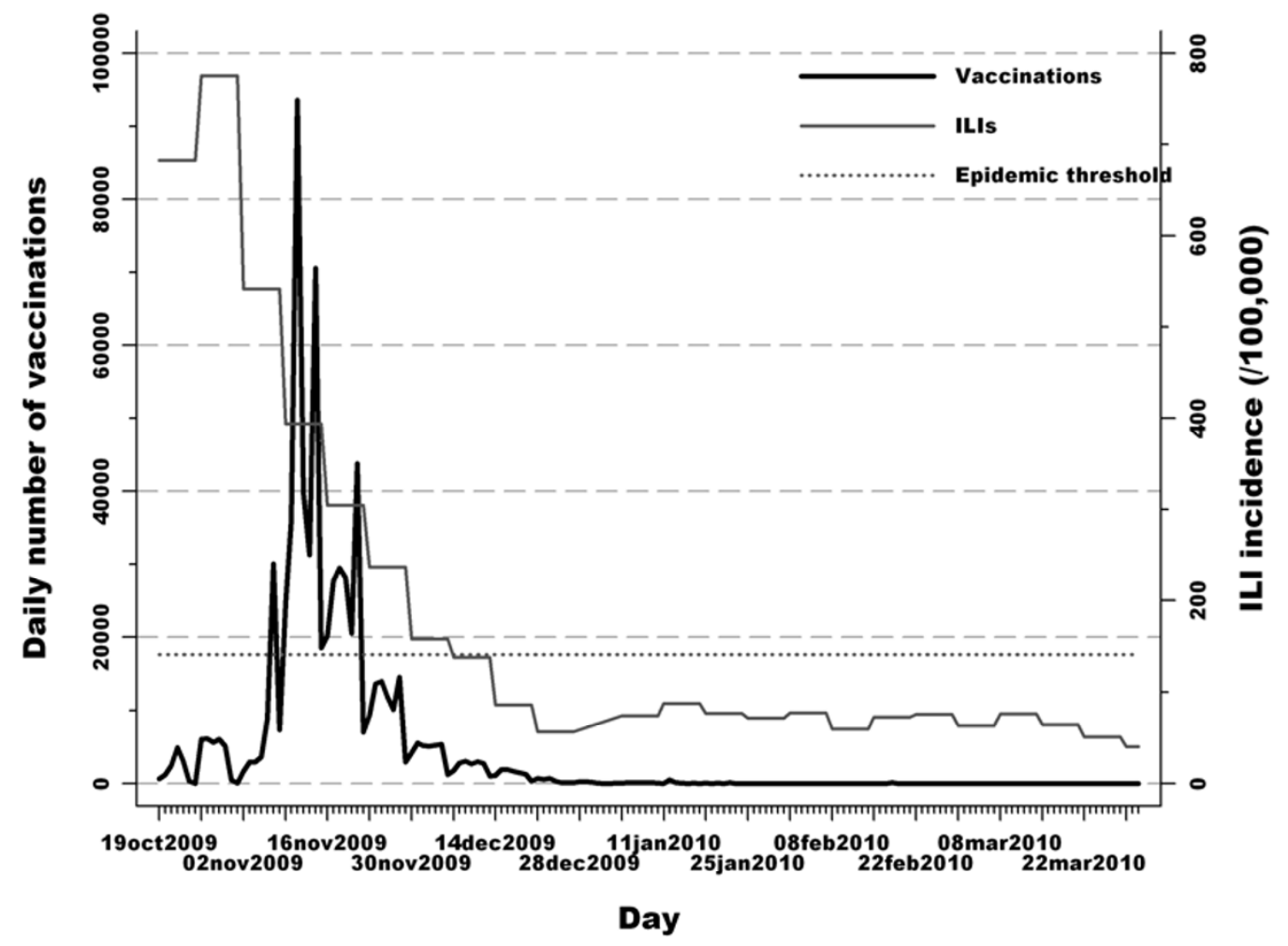

Vaccination coverage by region

The number of vaccinations recorded by region showed a big difference between Flanders and Wallonia/Brussels. Among all vaccination registrations, $87 \%$ occurred in the Flemish Region.

Figure 2 illustrates the cumulative percentage of vaccinations within each Belgian province. Flemish provinces (West-Vlaanderen, Oost-Vlaanderen, Antwerpen, Limburg and VlaamsBrabant) had a cumulative percentage between $8.7 \%$ and $11.3 \%$ and the Walloon provinces (Hainaut, Namur, Liège, Luxembourg, Brabant wallon) as well as the Brussels-Capital Region had a cumulative percentage between $1.3 \%$ and $1.9 \%$. These differences were due to a controversy among French-speaking practitioners. They felt uncomfortable about recording their vaccinations mainly for confidentiality reasons. 


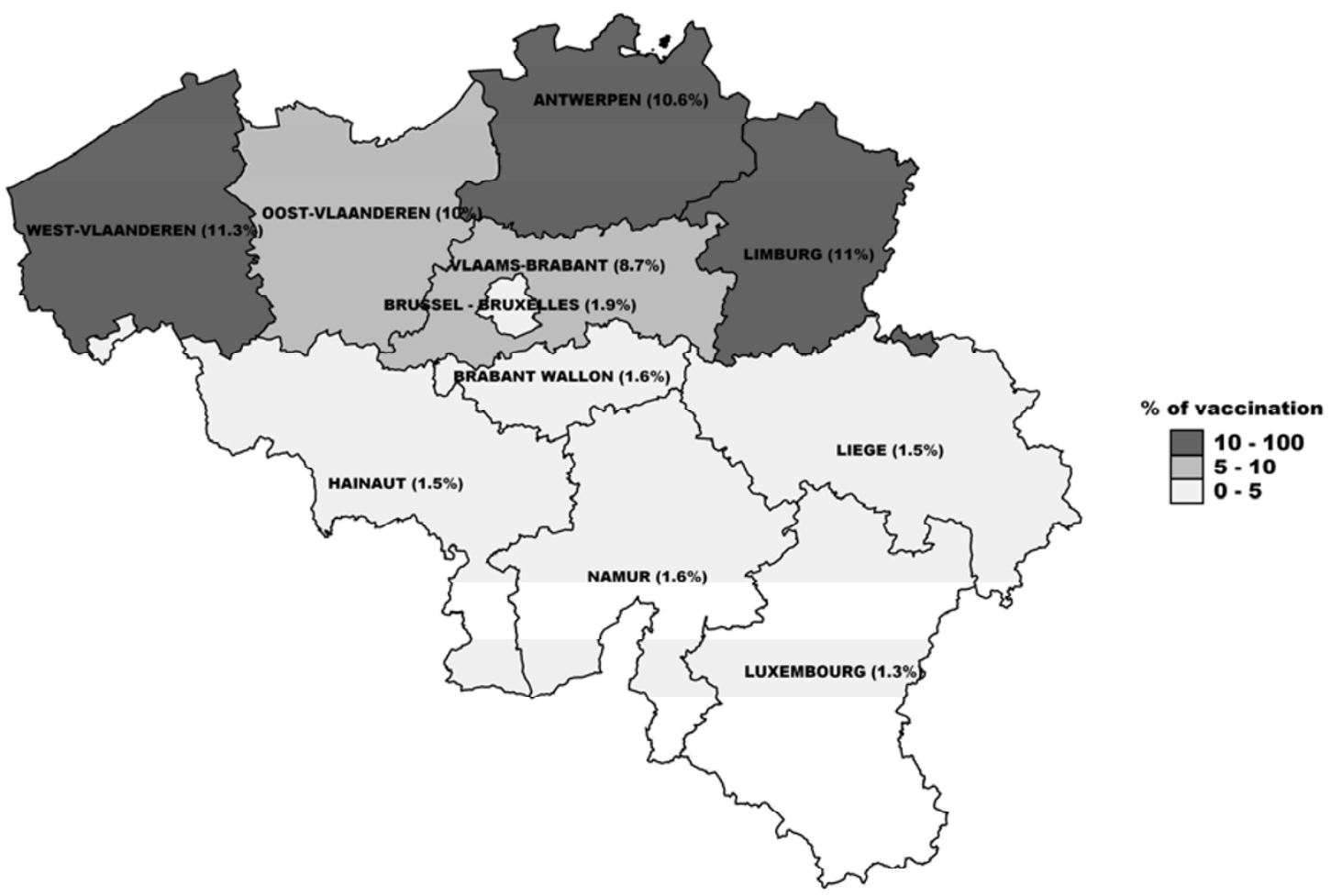

Vaccination coverage by age group

The vaccination coverage among age groups in Table 1 underlines that the elderly are the most vaccinated group with a coverage of $17.4 \%$ for the $65-84$-year-olds and $30.0 \%$ for the group of people aged 85 years and above. If the coverage is considered only for Flanders that has a better reporting, one quarter of the age group 65-84 years is vaccinated and $44 \%$ of the age group 85 years and more. Children (less than 14 years) are less vaccinated with around $1 \%$ of vaccination coverage. This situation corresponds to the authorities' recommendations as the elderly are the group with the highest risk that should be vaccinated due to pre-existing chronic pathologies.

Table 1. Reported vaccination coverage by age group, situation on 31 March 2010

\begin{tabular}{|l|l|l|l|l|}
\hline & \multicolumn{4}{|l|}{ Vaccination coverage $(\%)$ in } \\
\hline Age group & Belgium & Flanders & Wallonia & Brussels \\
\hline $0-4$ years & 0.8 & 1.1 & 0.2 & 0.2 \\
\hline $5-14$ years & 1.0 & 1.5 & 0.3 & 0.3 \\
\hline 15-64 years & 5.3 & 7.9 & 1.1 & 1.4 \\
\hline $65-84$ years & 17.4 & 24.9 & 3.9 & 5.2 \\
\hline 85 years + & 30.0 & 44.3 & 7.2 & 9.8 \\
\hline TOTAL & 6.56 & 10.2 & 1.5 & 1.9 \\
\hline
\end{tabular}




\section{Pharmacovigilance surveillance}

From 19 October until 15 February 2010, a total of 158 Adverse Drug Reaction (ADRs) reports have been sent to the Belgian Centre of Pharmacovigilance (BCP) of the FAMHP. All reports came from Health Care Professionals (physicians / pharmacists) or from GSK, the Marketing Authorisation Holder (MAH) of Pandemrix. The most frequently reported ADRs were injection site reactions, influenza-like illness including fever and myalgia, allergic reactions (rash, urticaria, bronchospasm, angiooedema and anaphylaxis) and neurological reactions (especially paraesthesia and neuritis). All these reactions are mentioned in the summary of product characteristics and in the public leaflet of Pandemrix. The BCP also registered 6 cases of deaths, related in time to the vaccination. In one case, the patient was a 30-year-old man who had a cardiac arrest. In the other cases, the age of the patients was between 68 and 90 years. For five of the six cases, important comorbidity confounding factors such as chronic cardiac and/or respiratory disease could explain the death. In case of the 30-year-old man, it was difficult to correctly assess this case in the absence of an autopsy. The link with the vaccination could not be ruled out as there is a temporal relation with the event. However, sudden deaths may occur in apparent healthy subjects $(8,9)$. The BCF did not receive any case of Guillain-Barré syndrome. The safety profile observed in Belgium is in line with the safety data reported at the European level (7).

A broad evaluation was made of the relative reporting rate in Belgium of the vaccine's ADRs compared to the total reporting rate for all medicinal products. This was done by comparing the number of ADR reports received for the pandemic vaccine (during the vaccination campaign) and for all medicinal products (on a yearly basis). The ratio obtained in Belgium was $28 \%$, compared to $18 \%$ in France and $13 \%$ in the United Kingdom. We can conclude that the relative reporting rate of ADRs for the pandemic vaccines was better in Belgium than in France and UK. Nevertheless, this estimation should be cautiously interpreted as the absolute number of ADRs reported, when corrected in function of the population, remains very low in Belgium (number of ADR reports per million inhabitants in Belgium: 16.6, in France: 60.3 and in UK: 52.2).

\section{Discussion}

Benefits of the system

This web-based recording system was easily accessible for general practitioners and citizens with Internet access and an identity card reader. The limited number of coding fields, the data validity control rules and the link with the national database, together with a short encoding phase, offer a significant amount of information of quality.

Information provided by the system was first of all of importance for the authorities to follow the vaccination coverage rate in the population. In case of an adverse effect of the vaccine, this database can also be very useful to identify vaccinated persons. In the pharmacovigi- 
lance point of view, exposure data are very important in order to make observed versus expected ADR analyses. Moreover, the age stratification of the exposure data could be useful in the context of massive children / elderly patients vaccination. The specific pharmacovigilance reporting system established in Belgium in order to control the safety of the pandemic vaccine was a success in view of the total rate of reporting for all medicinal products.

Weaknesses of the system

Authorities should improve the communication about the confidentiality of the information and should convince people, especially practitioners, of the interest of this kind of tool. This database can be really effective if a maximum of concerned persons would join the project. The recording system is still active to allow practitioners to record former vaccinations.

Without having more information about the proportion of chronic diseases in the different age groups, the vaccination coverage for targeted groups is difficult to estimate.

\section{References}

1. Influenza Scientific Committee. Fiche de vaccination grippe pandémique-Vaccin Pandemrix. Avis du 03/11/2009. http://www.influenza.be/fr/_documents/AvisVaccin03112009.pdf [accessed 23 march 2010]

2. World Health Organization. Vaccines for pandemic (H1N1) 2009. http://www.who.int/csr/disease/swineflu/frequently_asked_questions/vaccine_preparedness/en/index.ht $\mathrm{ml}$ [accessed 21 january 2010]

3. European Center for Disease Prevention and Control. General Q\&As about the pandemic vaccine. http://ecdc.europa.eu/en/healthtopics/H1N1/Pages/vaccines.aspx [accessed 21 january 2010]

4. Influenza Scientific Committee. Grippe A/H1N1: informations pour les citoyens. http://www.influenza.be/fr/H1N1_fr.asp [accessed 22 january 2010]

5. eHealth. Enregistrement de la vaccination $\mathrm{A} / \mathrm{H} 1 \mathrm{~N} 1$ https://www.ehealth.fgov.be/fr/application/applications/EH1N1VACC.html [accessed 22 january 2010]

6. Van Casteren V, Mertens K, Antoine J, Wanyama S, Thomas I, Bossuyt N. Clinical influenza surveillance of Influenza A(H1N1)2009 pandemic through the network of Sentinel General Practitioners. Arch Pub Health 2010; 68(2): 62-7

7. European Medicines Agency. Updates on pandemic safety monitoring. http://www.ema.europa.eu/influenza/updates.html [accessed 26 july 2010]

8. Danieli G A (2005). Sudden Arrhythmic Death: Which Genetic Determinants?. Cardiac Arrhythmias Proceedings of the 9th International Workshop on Cardiac Arrhythmias. A. Raviele. Venice 385-92

9. Behr E., Wood D.A., Wright M., Syrris P., Sheppard M.N., Casey A., Davies M.J., McKenna W. (2003). Cardiological assessment of first-degree relatives in sudden arrhythmic death syndrome. Lancet. 362: 1457-9 